\title{
DNA Profile Components Predict Malignant Outcomes in Select Cases of Intraductal Papillary Mucinous Neoplasm with Negative Cytology
}

Rachel E. Simpson MD ${ }^{a}$, Nathan J. Cockerill MD $^{a}$, Michele T. Yip-Schneider PhD ${ }^{a, b}$, Eugene P. Ceppa $M^{a, b}$, Michael G. House MD ${ }^{a}$, Nicholas J. Zyromski MDª, Attila Nakeeb MDa, Mohammad A. Al-Haddad $\mathrm{MD}^{\mathrm{b}, \mathrm{c}}$, C. Max Schmidt MD/MBA/PhD ${ }^{\mathrm{a}, \mathrm{b}, \mathrm{d}-\mathrm{f}}$

${ }^{\text {a }}$ Department of Surgery, Indiana University School of Medicine, Indianapolis, IN

${ }^{b}$ Indiana University Health Pancreatic Cyst and Cancer Early Detection Center, Indianapolis, IN

${ }^{c}$ Department of Medicine, Division of Gastroenterology, Indiana University Hospital, Indianapolis

${ }^{\mathrm{d}}$ Indiana University Simon Cancer Center, Indianapolis, IN

${ }^{\mathrm{e}}$ Walther Oncology Center, Indianapolis, IN

${ }^{\mathrm{f}}$ Department of Biochemistry/Molecular Biology, Indiana University School of Medicine, Indianapolis, IN

\section{Correspondence Address:}

C. Max Schmidt, MD, PhD, MBA

Department of Surgery

Indiana University School of Medicine

545 Barnhill Drive, Emerson Hall 129

Indianapolis, IN 46202

Phone: (317) 948-8358＜noBreak>＜noBreak>Email: maxschmi@iupui.edu

Presentation:

Selected for Oral Presentation at the Central Surgical Association annual meeting

Columbus, $\mathrm{OH}$ 3/15/18-3/17/18

\section{Financial Support:}

No external financial support or grant funding was used in this research study

\section{Conflict of Interest (All Authors):}

None

This is the author's manuscript of the article published in final edited form as:

Simpson, R. E., Cockerill, N. J., Yip-Schneider, M. T., Ceppa, E. P., House, M. G., Zyromski, N. J., ... \& Schmidt, C. M. (2018). DNA profile components predict malignant outcomes in select cases of intraductal papillary mucinous neoplasm with negative cytology. Surgery, 164(4), 712-718. https://doi.org/10.1016/j.surg.2018.05.033 


\section{ABSTRACT}

Background: Predicting malignancy in intraductal papillary mucinous neoplasm (IPMN) remains challenging. Integrated molecular pathology combines pancreatic fluid DNA and clinical factors into a malignant potential score. We sought to determine the utility of DNA components alone in predicting $H G D /$ Invasive disease.

Methods: We retrospectively reviewed records from 1106 patients with IPMN. We excluded non-IPMN cases, and cases with definitive malignant cytology. A total 225 patients had 283 DNA profiles (98 followed by surgery, 185 followed by $\geq 23$ month surveillance). HGD/Invasive outcomes were HGD, IPMN-invasive, and adenocarcinoma on surgical pathology or mesenteric/vascular invasion, metastases or biopsy with HGD or adenocarcinoma during surveillance.

Results: High Quantity DNA predicted $(\mathrm{P}=0.004) H G D /$ Invasive outcomes with sensitivity of 78.3\%, but 52.7\% specificity, indicating benign cases may exhibit High Quantity DNA. High clonality loss of heterozygosity (HC LOH) of tumor suppressor genes was $98.0 \%$ specific, strongly predicting $H G D /$ Invasive disease, but lacked sensitivity (20.0\%.) High Quantity DNA + HC LOH had $99.0 \%$ specificity for HGD/Invasive disease. KRAS mutation alone did not predict HGD/Invasive disease, but combined with High Quantity DNA (specificity 84.7\%) and HC LOH (specificity $99.0 \%$ ) strongly predicted $H G D /$ Invasive outcomes.

Conclusion: Certain DNA components are highly specific for $H G D$ /Invasive disease and may indicate aggressive lesions requiring resection when cytology fails. 
Abbreviations:

Carcinoembryonic Antigen (CEA)

High Clonality (HC)

High-grade or High-grade Dysplasia (HGD)

High-Risk Stigmata (HRS)

2012 International Consensus Guidelines (ICG)

Integrated molecular pathology (IMP)

Intraductal Papillary Mucinous Neoplasm (IPMN)

Loss of Heterozygosity (LOH)

Negative Predictive Value (NPV)

Positive Predictive Value (PPV)

Worrisome Features (WF) 


\section{INTRODUCTION}

Intraductal papillary mucinous neoplasms (IPMN) are one of the few known premalignant lesions of the pancreas, and have been diagnosed with increasing frequency. When first described in the 1980's by Ohashi et al., IPMN were felt to be incredibly rare. ${ }^{1}$ However, others have concluded IPMN to be one of the most common pancreatic cystic neoplasm, representing $24 \%$ of cysts resected at a single institution. ${ }^{2}$ Thus, the tremendous effort dedicated to understanding IPMN over the preceding decades has been warranted.

IPMN may transform through progressive grades of dysplasia, from low, moderate, highgrade (HGD), to invasive IPMN. Current 2012 International Consensus Guidelines (ICG) focus on clinical and radiographic criteria to predict the most aggressive lesions necessitating resection depending on the presence of High-Risk Stigmata (HRS) or Worrisome Features (WF). ${ }^{3}$ These consensus guidelines have been noted to provide reasonable sensitivity (72-90\%) but often poor specificity (46-78\%) for detecting HGD or invasive IPMN. ${ }^{4}{ }^{5}$ For this reason, research dedicated to understanding IPMN at a molecular level has gained favor.

Since the mid-2000's, PancraGEN from Interpace Diagnostics (previously Redpath) has sought to predict which pancreatic cysts held the highest potential for malignancy. Pancreatic cyst or ductal fluid gathered via endoscopy is analyzed for level and quality of DNA, oncogene mutations and loss of heterozygosity (LOH) of tumor suppressor genes. Clinical data (cyst size, main pancreatic duct diameter, cyst fluid Carcinoembryonic Antigen (CEA) level) is combined with these DNA factors to develop an aggregate malignancy risk score. This testing process is

termed Integrated Molecular Pathology (IMP). ${ }^{6}$ However, this integration process makes it difficult to determine the merits of the DNA components alone. 
Prior studies have sought to determine if DNA features could predict mucinous differentiation as well as malignancy. Several studies have proposed High Quantity and/or High Quality DNA on pancreatic cyst fluid analysis as a predictor of a non-benign or malignant course of pancreatic cysts. ${ }^{7-11}$ Mutations of the KRAS oncogene have been studied extensively, and are commonly discovered in pancreatic and other cancers; $;{ }^{12}$ but results of prior studies regarding the ability to predict a malignant outcome for pancreatic cysts are conflicting. ${ }^{8,10-15}$ The presence of KRAS or GNAS mutations have been determined to be specific for mucinous differentiation of pancreatic cysts, ${ }^{8,} 12,15-17$ with GNAS especially specific for IPMN. ${ }^{18,} 19$ Allelic LOH of tumor suppressor genes has also been associated with mucinous differentiation or malignant pathology, ${ }^{17}$, ${ }^{20}$ especially when in combination with KRAS mutations. ${ }^{7-11,13,21}$

Studies dedicated to IPMN, especially the surveillance population, are lacking, making the application of prior findings to this specific population unclear. Our study focuses on the predictive capabilities of each DNA component for patients with IPMN specifically, including both surgical and surveillance cohorts.

\section{METHODS}

Patient Selection: A prospectively maintained database of patients with IPMN ( $\mathrm{n}=1106)$ from Indiana University was retrospectively reviewed. We queried for patients with non-malignant cytology (no definitive HGD or carcinoma) on endoscopic ultrasound guided fine-needle aspiration (EUS-FNA) of cyst/duct fluid that had subsequent IMP testing performed. 283 IMP cases (225 patients) were examined: 98 cases were followed by surgery whereas 185 were followed by a minimum of 23 months surveillance. The surgical cohort was defined by IPMN on surgical pathology specimens corresponding to the anatomic location of cyst fluid analysis. Inclusion in the surveillance cohort required convincing evidence of IPMN, i.e., multifocal pancreatic cysts; 
solitary pancreatic cysts with a connection to the main pancreatic duct or main duct dilation with cytology suggestive of IPMN, cyst fluid mucin, CEA $>192 \mathrm{ng} / \mathrm{mL}$ or GNAS mutation.

A HGD/invasive outcome included HGD/invasive IPMN or adenocarcinoma on surgical pathology; malignant cytology on subsequent biopsy; and radiographic/endoscopic evidence of invasive mass lesion or metastatic disease during surveillance.

At our institution, patients diagnosed with pancreatic cysts $>1 \mathrm{~cm}$ or main duct dilation not clearly representative of IPMN typically undergo initial EUS-FNA with DNA profiling to establish a baseline diagnosis based on endoscopic appearance, fluid and cytology characteristics, and molecular profile. Patients undergoing surveillance are recommended for subsequent testing every 3 or 5 years based on stratification to a high- or low-risk protocol respectively. A total $200 \mu \mathrm{L}$ of fluid are optimal for testing, and coverage varies by insurance.

Clinically, patients were selected for surgery or surveillance primarily based on the presence/absence of symptoms, general health and surgical candidacy, progression of disease, cytology, and ICG criteria. As the role of DNA profiling is not entirely clear for the IPMN population, this data was not used independently to recommend a management strategy.

Integrated Molecular Pathology Molecular Parameters: In our study, patients with any value besides Low Quantity DNA (range Low-Greatly Elevated) were considered to have "High Quantity” DNA. Similarly, patients with all but Poor Quality DNA (range Poor-Good) were designated to the "High Quality" DNA group. "High Risk” DNA designation requires both High Quantity and High Quality DNA. Oncogene mutations (KRAS and GNAS) and allelic LOH are also reported. ${ }^{22}$ These are considered High Clonality (HC) when $\geq 75 \%$ of the DNA is affected. ${ }^{23}$ Statistical Analysis: The sensitivity, specificity, accuracy, positive predictive value (PPV), negative predictive value (NPV) and positive likelihood ratios (LR+) were calculated. Chi-square 
analysis/Fisher's Exact test were used to determine significant associations ( $\alpha$-level of 0.05.) IBM SPSS software, version 24 was used for these analyses.

\section{RESULTS}

Surgery and Surveillance Cohorts: Benign vs. HGD/Invasive: Of the 283 IMP cases (225 patients), 98 were followed by pancreatectomy, while the remaining 185 were followed by a minimum of 23 months surveillance. Twenty-three IMP cases were associated with a HGD/Invasive outcome determined by surgical pathology, with a median time to diagnosis of 5.2 months after IMP. The other 260 cases had a benign outcome, concluded by surgical pathology or surveillance at a median 3.6 months and 47.3 months after IMP respectively. (Table 1)

The most sensitive predictor of $H G D /$ Invasive outcome was the presence of High Quantity DNA (sensitivity $78.3 \%, \mathrm{P}=0.004$ ). However, High Quantity DNA alone was poorly specific (52.7\%), and not an accurate (54.8\%) predictor of $H G D /$ Invasive disease, with low PPV (12.8\%). Thus, most HGD/Invasive lesions exhibit High Quantity DNA, but many benign lesions exhibit this feature as well. The single most specific DNA factor for HGD/Invasive disease was the presence of $\mathrm{HC} \mathrm{LOH}$ (specificity $98.0 \%, \mathrm{P}=0.003$ ). Alone, $\mathrm{HC} \mathrm{LOH}$ had PPV of $50 \%$, but occurred proportionally much more frequently in the $H G D /$ Invasive group with a LR+ of 10 . The combination of High Quantity DNA and HC LOH $(\mathrm{P}=0.006)$ provided even greater specificity (99.0\%), accuracy (91.4\%), PPV (60\%), and LR+ (15). An LR+ of this value provides a post-test probability of $H G D /$ Invasive disease tremendously greater than the pre-test probability, and strongly suggests the possibility of more aggressive disease. The combination of High Quality DNA or High Risk DNA and HC LOH provided $100 \%$ specificity, $>90 \%$ accuracy, and a PPV of $100 \%(\mathrm{P}<0.05)$. HC LOH in addition to a HC KRAS mutation was associated with $H G D /$ Invasive outcomes ( $\mathrm{P}=0.011)$, with a specificity of $98.5 \%$ and accuracy of $90.9 \%$. The presence of both 
KRAS and GNAS mutations, in addition to elevated Quantity and/or Quality of DNA was significantly associated with $H G D /$ Invasive disease $(\mathrm{P}=0.032)$ with moderate sensitivity $(66.7 \%)$, high specificity (95.2\%) and accuracy (91.7\%), and reasonable PPV (66.7\%). DNA components reaching/approaching significant associations with $H G D /$ Invasive disease are summarized in Table 2.

Several other DNA components, alone and in combination, revealed high specificity for $H G D /$ Invasive outcomes, but statistical or near-statistical significance on Chi-square analysis was not reached. These are shown in Supplemental Table 1s (for the online version).

Surgery Cohort Alone: Benign vs. HGD/Invasive: 98 IMP cases were followed by surgery, with a resulting $23 H G D /$ Invasive and 75 benign outcomes. High Quantity DNA $(\mathrm{P}=0.019)$ again was the most sensitive (78.3\%) in determining $H G D /$ Invasive disease; however, specificity (49.3\%), accuracy (56.1\%), and PPV (32.1\%) were overall poor. High Quality DNA ( $\mathrm{P}=0.019)$ and High Risk DNA $(\mathrm{P}=0.015)$ were less sensitive $(47.8 \%$ and $43.5 \%)$, but more specific $(77.3 \%$ and 81.3\%), with greater accuracy (70.4\% and 72.4\%) and slightly improved PPV $(39.3 \%$ and $41.7 \%)$ for $H G D$ /Invasive lesions. The combination of High Quality DNA with $\mathrm{HC}$ LOH $(\mathrm{P}=0.017)$ provided the greatest specificity (100.0\%), accuracy $(77.3 \%)$ and PPV (100\%). However, this combination occurs rarely, thus lacked sensitivity (15.0\%). In contrast to the surgery + surveillance cohort, HC LOH alone fell short of statistical significance $(\mathrm{P}=0.077)$, but continued to show high specificity (94.5\%) and accuracy (74.7\%) for HGD/Invasive outcomes, with a PPV of 57.1\%. HC LOH in combination with High Risk DNA provided even greater specificity (100.0\%), accuracy (76.0\%), and PPV (100\%), but did not reveal a significant relationship with HGD/Invasive outcomes $(\mathrm{P}=0.068)$. Any degree of elevated DNA (High Quantity +/- High Quality) in addition to both KRAS and GNAS mutation most closely approximated a statistically significant 
relationship with $H G D /$ Invasive disease ( $\mathrm{P}=0.056)$, with fair sensitivity $(66.7 \%)$, good specificity (93.3\%), moderate PPV (66.7\%), and the highest accuracy of any combination (88.9\%). Furthermore, this combination of DNA components provided the highest $\mathrm{LR}+$ of 10 , which greatly increases the post-test probability of $H G D /$ Invasive disease when present. (Table 3).

Several other DNA components and combinations showed high specificity for $H G D /$ Invasive disease, but no statistically significant relationship. These are outlined in Supplemental Table 2s (for the online version).

Outcome Predictions Based on 2012 Consensus Guidelines Criteria: When considering all HRS, WF (excluding pancreatitis), and EUS findings, patients recommended for surgery based on ICG (ICG+, $\mathrm{n}=44$ ) had a rate of $H G D /$ Invasive disease of $29.5 \%$. When patients from the ICG+ group with HRS were excluded to include only patients with WF, or more "borderline" lesions, (WF+, $\mathrm{n}=111$ ), the rate of $H G D /$ Invasive disease was $9.9 \%$. Finally, patients lacking HRS or WF (ICG-, $\mathrm{n}=158$ ) had the lowest rate of $H G D /$ Invasive disease at $1.9 \%$. Separate analyses were performed on these strata. In ICG+ or WF+ cases, $\mathrm{HC}$ LOH was significantly associated with $H G D /$ Invasive disease ( $\mathrm{P}<0.05)$, with high specificity $(>98.0 \%)$ and moderate accuracy $(>77 \%)$, with PPV of $100 \%(\mathrm{ICG}+)$ and $66.7 \%(\mathrm{WF}+)$. HC LOH plus High Quality DNA ( $\mathrm{P}=0.120)$ or HC KRAS $(\mathrm{P}=0.013)$ mutation in the $\mathrm{WF}+$ group provided $100 \%$ specificity and $100 \% \mathrm{PPV}$. The ICG- group revealed no statistically significant relationships. The most compelling DNA feature was High Quantity DNA ( $\mathrm{P}=0.118)$, with great sensitivity $(100 \%)$, but poor specificity $(51.6 \%)$ and accuracy (52.5\%). Though the PPV of this DNA feature was poor (3.9\%), the NPV reached $100 \%$. These and other DNA analyses are summarized in Table 4. 


\section{DISCUSSION}

IPMN are known to harbor malignant potential, but differentiating between low and higher risk lesions is difficult. In the era of individualized medicine, it is no surprise that the genetic profile of these cysts will be increasingly incorporated into clinical decision-making. Prior studies have described the utility of cyst fluid DNA in predicting mucinous differentiation or malignancy of pancreatic cysts in general. To our knowledge, the present study is the largest evaluation of the value of the DNA components for the IPMN-specific surgical and surveillance population.

We found the most sensitive determinant of a HGD/Invasive outcome was the presence of High Quantity DNA (78.3\%). This finding is mechanistically plausible, as the development of cancer relies on the unregulated, disorganized growth and proliferation of cells. ${ }^{24}$ However, as Khalid et al. describes, certain benign processes, such as inflammation or necrosis, may lead to the release of cellular DNA. ${ }^{9}$ This may explain why, though highly sensitive, High Quantity DNA is poorly specific for $H G D /$ Invasive disease in our surgical +/- surveillance analyses. High Quality DNA was a predictor of $H G D /$ Invasive disease in the surgery alone group, with greater specificity $(>75 \%)$. DNA may degrade over time in a stagnant cyst or in the presence of a benign inflammatory process. Therefore, in the presence of rapid proliferation and absence of benign inflammatory disease, the presence of High Quality DNA may suggest a malignant processes.

Most prior studies examining the role of KRAS mutation in predicting $H G D /$ Invasive pathology in all pancreatic cyst types have revealed no significant association. ${ }^{8,10,12}$ In the present study, we validate these prior findings in our IPMN-specific population, as we found no significant relationship between KRAS mutation and $H G D /$ Invasive outcomes in our surgery +/- surveillance analyses. KRAS mutation only revealed predictive potential for $H G D /$ Invasive disease in the presence of concomitant mutations or elevated Quantity or Quality of DNA. 
The presence of $\mathrm{HC} \mathrm{LOH}$ was the most consistent predictor of $H G D /$ Invasive outcomes in the present study. HC LOH alone was highly specific for HGD/Invasive IPMN (98.0\%) in our analysis of surgery + surveillance patients. However, when surgical patients were examined alone, though still highly specific, the association only approached statistical significance. Schoedel et al. and Khalid et al. have previously shown an association between KRAS mutations $+\mathrm{LOH}$ and $H G D /$ Invasive outcomes. ${ }^{10,21}$ Similarly, we demonstrated a significant association between $\mathrm{HC}$ $\mathrm{LOH}+\mathrm{KRAS}$ mutation and $H G D /$ Invasive outcomes in our surgery + surveillance analysis. In our surgical cohort, the combination of HC LOH and High Quality DNA provided 100\% specificity for $H G D /$ Invasive outcomes. To our knowledge this has not been documented prior in the literature.

In our subgroub analysis of ICG+, $\mathrm{WF}^{+}$, and ICG- patients, we found that the ability of DNA features to predict $H G D /$ Invasive disease varied, likely owed to differing prevalence of $H G D /$ Invasive outcomes in these strata. In practice, patients that are ICG+ are frequently recommended for surgery. However, not all individuals are amenable or optimal surgical candidates. In the ICG + and $\mathrm{WF}+$ subgroups, $\mathrm{HC}$ LOH again was highly specific for $H G D /$ Invasive disease, with or without the presence of High Quality DNA or KRAS mutation, with a PPV $66.7-100 \%$. Therefore, the presence of $\mathrm{HC} \mathrm{LOH}$ in patients that are ICG+ but not optimal surgical candidates, or have more borderline lesions (WF+) should alert the clinical to a greater likelihood of HGD/Invasive disease. For those lacking any HRS or WF (ICG-), the presence of High Quantity DNA provided $100 \%$ sensitivity with a NPV of $100 \%$. Thus, the absence of High Quantity DNA only occurred in benign cases. Though this did not reach statistical significance, one may consider the absence of High Quantity DNA to support ongoing or 
potentially relaxed surveillance over surgery in this group. As the numbers in these subgroups were small, future studies are needed to validate these findings.

This study includes a specific subgroup of patients with IPMN who have undergone EUSFNA with resulting non-malignant cytology and subsequent IMP testing. Cytology historically has shown high specificity but low sensitivity $(65 \%)$ for detecting malignant IPMN. ${ }^{25}$ Our data in combination with prior studies demonstrates that DNA profiling may be useful in capturing more $H G D /$ Invasive IPMN when the gold standard of cytology fails.

Our study had several potential limitations. The number of HGD/Invasive cases is relatively small. This is in part due to the exclusion of patients with preoperative cytology or core biopsy revealing high grade atypia or adenocarcinoma for whom DNA profiling is not performed, as it would not enhance clinical decision making. Thus, blatantly malignant or aggressive lesions on imaging or by EUS-FNA cytology tend to either immediately undergo surgery or are selfselected out of the IMP testing process. Because of this, the power of our study may be limited. Molecular pathology and surveillance outcome data was gathered retrospectively to supplement our prospectively maintained IPMN database. It is plausible that a fraction of our surveillance cohort may contain non-IPMN cystic lesions. While surgical pathology, cytology, or biopsy would ideally serve as the gold standard for diagnosis, patients without these diagnostic tests have lessdefinitive outcomes. We attempted to minimize the potential for misallocation to our benign vs $H G D / I n v a s i v e$ groups through exclusion of patients with insufficient duration of follow-up $(<23$ months) and imaging surveillance (radiographic or endoscopic) available at this 23 month point. Finally, the number of patients with specific DNA parameters is oftentimes small, and should be interpreted cautiously. 
The present study is unique in a number of ways. We performed the largest evaluation of cyst fluid DNA components on an IPMN-specific population to date. This helps to provide internal validity for the application of these DNA parameters to IPMN specifically, rather than pancreatic cysts in general. Most prior studies exclude surveillance patients; the inclusion of non-surgical patients in our study we feel is more representative of the true IPMN population. However, surgical pathology provides the most definitive means of determining a HGD/Invasive or benign outcome. Therefore, the DNA measures with the strongest predictive power are likely to be reflected in the surgery population.

We proposed several DNA profiles that may aid the clinician in decision making, especially in cases of non-diagnostic cytology. The majority of HGD/Invasive IPMN exhibit High Quantity of DNA, and can be considered the best candidate for a "screen" for this more advanced disease. However, this feature is nonspecific, as many benign cysts also exhibit elevated levels of DNA. HC LOH alone and in combination with other DNA factors is most consistently associated with $H G D$ /Invasive outcomes with a high degree of specificity, and should alert the physician to a higher probability of aggressive disease requiring resection. KRAS mutations only predicted HGD/Invasive IPMN when combined with other DNA factors. Thus, the predictive role of KRAS mutation is less clear. Molecular profiling of pancreatic cyst fluid should not be used as a standalone test, but can be useful as an adjunct to established diagnostic testing. 


\section{TABLES/FIGURES}

Table 1. Descriptive Statistics for HGD/Invasive Outcomes (HGD, PDAC, IPMN-Invasive)

Surgery $(n=98) \quad$ Surveillance $(n=185)$

Benign $\quad 75(3.6 ; 2.1-11.4$ months $) \quad 185$ (47.3; 35.6-63.2months)

HGD/Invasive $23(5.2 ; 1.3-14.6$ months $) \quad 0$

HGD High-Grade IPMN; PDAC Pancreatic Ductal Adenocarcinoma; N (Time to outcome determination: Median; Interquartile Range); Time based on time of surgery, or most recent benign follow-up 
Table 2. Most Significant Predictors of HGD/Invasive Outcome: Surgery and Surveillance

\begin{tabular}{|c|c|c|c|c|c|c|c|c|c|}
\hline & TP & FP & $\begin{array}{c}\text { Sn } \\
(\%)\end{array}$ & $\begin{array}{c}\text { Sp } \\
(\%)\end{array}$ & $\begin{array}{l}\text { Acc } \\
(\%)\end{array}$ & LR+ & $\begin{array}{l}\text { PPV } \\
\text { (\%) }\end{array}$ & $\begin{array}{l}\text { NPV } \\
(\%)\end{array}$ & P-Value \\
\hline High Quantity DNA & $(18 / 23)$ & $(123 / 260)$ & 78.3 & 52.7 & 54.8 & 2 & 12.8 & 96.5 & $0.004 *$ \\
\hline$+K R A S$ & $(8 / 22)$ & $(36 / 235)$ & 36.4 & 84.7 & 80.5 & 2 & 18.2 & 93.4 & $0.032 *$ \\
\hline$+K R A S+G N A S$ & $(2 / 3)$ & $(1 / 21)$ & 66.7 & 95.2 & 91.7 & 14 & 66.7 & 95.2 & $0.032 *$ \\
\hline$+K R A S+H C L O H$ & $(2 / 20)$ & $(2 / 200)$ & 10.0 & 99.0 & 90.9 & 10 & 50.0 & 91.7 & $0.042 *$ \\
\hline$+H C L O H$ & $(3 / 20)$ & $(2 / 200)$ & 15.0 & 99.0 & 91.4 & 15 & 60.0 & 92.1 & $0.006 *$ \\
\hline High Quality DNA & $(11 / 23)$ & $(78 / 260)$ & 47.8 & 70.0 & 68.2 & 2 & 12.4 & 93.8 & 0.078 \\
\hline$+K R A S$ & $(5 / 22)$ & $(21 / 235)$ & 22.7 & 91.1 & 85.2 & 3 & 19.2 & 92.6 & 0.056 \\
\hline$+K R A S+G N A S$ & $(2 / 3)$ & $(1 / 21)$ & 66.7 & 95.2 & 91.7 & 14 & 66.7 & 95.2 & $0.032 *$ \\
\hline$+K R A S+H C L O H$ & $(2 / 20)$ & $(0 / 200)$ & 10.0 & 100.0 & 91.8 & - & 100.0 & 91.7 & $0.008 *$ \\
\hline$+H C L O H$ & $(3 / 20)$ & $(0 / 200)$ & 15.0 & 100.0 & 92.3 & - & 100.0 & 92.2 & $0.001 *$ \\
\hline$+\mathrm{LOH}$ & $(3 / 20)$ & $(7 / 200)$ & 15.0 & 96.5 & 89.1 & 4 & 30.0 & 91.9 & 0.051 \\
\hline High Risk DNA & $(10 / 23)$ & $(60 / 260)$ & 43.5 & 76.9 & 74.2 & 2 & 14.3 & 93.9 & $0.030^{*}$ \\
\hline$+K R A S$ & $(4 / 22)$ & $(15 / 235)$ & 18.2 & 93.6 & 87.2 & 3 & 21.1 & 92.4 & 0.066 \\
\hline$+K R A S+G N A S$ & $(2 / 3)$ & $(1 / 21)$ & 66.7 & 95.2 & 91.7 & 14 & 66.7 & 95.2 & $0.032 *$ \\
\hline$+H C L O H$ & $(2 / 20)$ & $(0 / 200)$ & 10.0 & 100.0 & 91.8 & - & 100.0 & 91.7 & 0.008* \\
\hline HC LOH & $(4 / 20)$ & $(4 / 200)$ & 20.0 & 98.0 & 90.9 & 10 & 50.0 & 92.5 & $0.003 *$ \\
\hline$+K R A S$ & $(3 / 20)$ & $(4 / 200)$ & 15.0 & 98.0 & 90.5 & 7 & 42.9 & 92.0 & $0.018^{*}$ \\
\hline$+H C K R A S$ & $(3 / 20)$ & $(3 / 200)$ & 15.0 & 98.5 & 90.9 & 10 & 50.0 & 92.1 & $0.011^{*}$ \\
\hline
\end{tabular}


Table 3. Most Significant Predictors of HGD/Invasive Outcome: Surgery Only

\begin{tabular}{lccccccccc}
\hline & TP & FP & $\begin{array}{c}\text { Sn } \\
(\%)\end{array}$ & $\begin{array}{c}\text { Sp } \\
(\%)\end{array}$ & $\begin{array}{c}\text { Acc } \\
(\%)\end{array}$ & LR+ & $\begin{array}{c}\text { PPV } \\
(\%)\end{array}$ & $\begin{array}{c}\text { NPV } \\
\text { (\%) }\end{array}$ & P-Value \\
\hline High Quantity DNA & $(18 / 23)$ & $(38 / 75)$ & 78.3 & 49.3 & 56.1 & 2 & 32.1 & 88.1 & $0.019^{*}$ \\
+KRAS + GNAS & $(2 / 3)$ & $(1 / 15)$ & 66.7 & 93.3 & 88.9 & 10 & 66.7 & 93.3 & 0.056 \\
High Quality DNA & $(11 / 23)$ & $(17 / 75)$ & 47.8 & 77.3 & 70.4 & 2 & 39.3 & 82.9 & $0.019^{*}$ \\
+KRAS +GNAS & $(2 / 3)$ & $(1 / 15)$ & 66.7 & 93.3 & 88.9 & 10 & 66.7 & 93.3 & 0.056 \\
+KRAS +HC LOH & $(2 / 20)$ & $(0 / 55)$ & 10.0 & 100.0 & 76.0 & - & 100.0 & 75.3 & 0.068 \\
+HC LOH & $(3 / 20)$ & $(0 / 55)$ & 15.0 & 100.0 & 77.3 & - & 100.0 & 76.4 & $0.017^{*}$ \\
High Risk DNA & $(10 / 23)$ & $(14 / 75)$ & 43.5 & 81.3 & 72.4 & 2 & 41.7 & 82.4 & $0.015^{*}$ \\
+KRAS +GNAS & $(2 / 3)$ & $(1 / 15)$ & 66.7 & 93.3 & 88.9 & 10 & 66.7 & 93.3 & 0.056 \\
+HC LOH & $(2 / 20)$ & $(0 / 55)$ & 10.0 & 100.0 & 76.0 & - & 100.0 & 75.3 & 0.068 \\
HC LOH & $(4 / 20)$ & $(3 / 55)$ & 20.0 & 94.5 & 74.7 & 4 & 57.1 & 76.5 & 0.077
\end{tabular}

TP True Positive (n-positive/n-tested in malignant group); FP False Positive (n-positive/n-tested in benign group); $S n$ Sensitivity; Sp Specificity; Acc Accuracy; LR+ Positive Likelihood Ratio; PPV Positive Predictive Value; NPV Negative Predictive Value; HC LOH High Clonality Loss of Heterozygosity of Tumor Suppressor Genes; High Quantity/Quality DNA = High Risk DNA; *denotes $P<0.05$ 
Table 4. Performance of DNA Features for Predicting HGD/Invasive Outcomes by Consensus Guidelines Subgroups

\begin{tabular}{|l|l|l|l|r|}
\hline P-value & Sensitivity (\%) & Specificity (\%) & Accuracy (\%) & LR+ \\
\hline
\end{tabular}

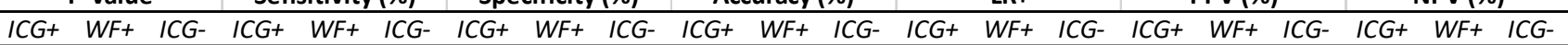

\begin{tabular}{|c|c|c|c|c|c|c|c|c|c|c|c|c|c|c|c|c|c|c|c|c|c|}
\hline High Quantity DNA & 0.376 & 0.113 & 0.118 & 69.2 & 72.7 & 100.0 & 45.2 & 55.0 & 51.6 & 52.3 & 56.8 & 52.5 & 1.3 & 1.6 & 2.1 & 34.6 & 15.1 & 3.9 & 77.8 & 94.8 & 100.0 \\
\hline High Quality DNA & 0.071 & 0.484 & 1.000 & 61.5 & 36.4 & 33.3 & 67.7 & 74.0 & 68.4 & 65.9 & 70.3 & 67.7 & 1.9 & 1.4 & 1.1 & 44.4 & 13.3 & 2.0 & 80.8 & 91.4 & 98.2 \\
\hline KRAS & 0.295 & 0.745 & 1.000 & 58.3 & 45.5 & 33.3 & 60.0 & 62.5 & 63.4 & 59.5 & 60.6 & 62.8 & 1.5 & 1.2 & 0.9 & 41.2 & 13.2 & 1.9 & 75.0 & 90.2 & 97.8 \\
\hline GNAS & 1.000 & 1.000 & - & 66.7 & 0.0 & - & 50.0 & 66.7 & 85.7 & 54.5 & 61.5 & 85.7 & 1.3 & 0.0 & - & 33.3 & 0.0 & 0.0 & 80.0 & 88.9 & 100.0 \\
\hline LOH & 0.358 & 0.108 & 1.000 & 30.0 & 20.0 & 0.0 & 85.7 & 95.9 & 87.8 & 67.7 & 86.7 & 85.7 & 2.1 & 4.9 & 0.0 & 50.0 & 40.0 & 0.0 & 72.0 & 89.7 & 97.3 \\
\hline HC LOH & 0.027 & 0.037 & 1.000 & 30.0 & 20.0 & 0.0 & 100.0 & 98.6 & 97.6 & 77.4 & 89.2 & 95.2 & - & 14.6 & 0.0 & 100.0 & 66.7 & 0.0 & 75.0 & 90.0 & 97.6 \\
\hline +High Qualit & 0.027 & 0.120 & - & 30.0 & 10.0 & 0.0 & 100.0 & 100.0 & 100.0 & 77.4 & 89.2 & 97.6 & - & 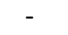 & - & 100.0 & 100.0 & - & 75.0 & 89.0 & 97.6 \\
\hline$+K R A S$ & 0.097 & 0.037 & 1.000 & 20.0 & 20.0 & 0.0 & 100.0 & 98.6 & 97.6 & 74.2 & 89.2 & 95.2 & 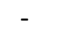 & 14.6 & 0.0 & 100.0 & 66.7 & 0.0 & 74.2 & 90.0 & 97.6 \\
\hline$+H C K R A S$ & 0.097 & 0.013 & 1.000 & 20.0 & 20.0 & 0.0 & 100.0 & 100.0 & 97.6 & 74.2 & 90.4 & 95.2 & . & - & 0.0 & 100.0 & 100.0 & 0.0 & 72.4 & 90.1 & 97.6 \\
\hline
\end{tabular}

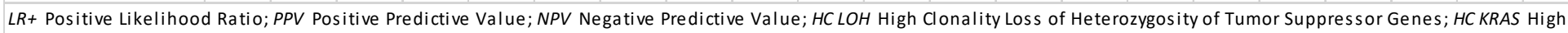

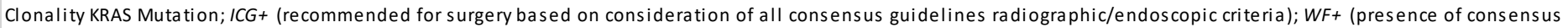
guidelines Worrisome Features only); ICG- (not recommended for surgery based on consideration of all consensus guidelines radiographic/endoscopic criteria) 
Table 1s. DNA Components Not Reaching Statistical Significance for HGD/Invasive Outcome:

Surgery and Surveillance

\begin{tabular}{|c|c|c|c|c|c|c|c|c|c|}
\hline & TP & FP & $\begin{array}{l}\text { Sn } \\
(\%)\end{array}$ & $\begin{array}{c}\text { Sp } \\
(\%)\end{array}$ & $\begin{array}{l}\text { Acc } \\
(\%)\end{array}$ & LR+ & $\begin{array}{r}\text { PPV } \\
(\%)\end{array}$ & $\begin{array}{l}\text { NPV } \\
\text { (\%) }\end{array}$ & $\begin{array}{c}\text { P- } \\
\text { Value }\end{array}$ \\
\hline High Quantity DNA & - & - & - & - & - & - & - & - & - \\
\hline$+H C$ KRAS & $(3 / 22)$ & $(12 / 235)$ & 13.6 & 94.9 & 87.9 & 3 & 20.0 & 92.2 & 0.126 \\
\hline +GNAS & $(2 / 3)$ & $(4 / 21)$ & 66.7 & 81.0 & 79.2 & 4 & 33.3 & 94.4 & 0.143 \\
\hline$+H C$ GNAS & $(1 / 3)$ & $(1 / 21)$ & 33.3 & 95.2 & 87.5 & 7 & 50.0 & 95.9 & 0.239 \\
\hline$+\mathrm{LOH}$ & $(3 / 20)$ & $(12 / 200)$ & 15.0 & 94.0 & 86.8 & 3 & 20.0 & 91.7 & 0.144 \\
\hline$+K R A S+G N A S+L O H$ & $(1 / 3)$ & $(1 / 18)$ & 33.3 & 94.4 & 85.7 & 6 & 50.0 & 89.5 & 0.271 \\
\hline$+K R A S+L O H$ & $(1 / 20)$ & $(5 / 200)$ & 5.0 & 97.5 & 89.1 & 2 & 16.7 & 91.1 & 0.439 \\
\hline High Quality DNA & - & - & - & - & - & - & - & - & - \\
\hline$+H C K R A S$ & $(2 / 22)$ & $(8 / 235)$ & 9.1 & 96.6 & 89.1 & 3 & 20.0 & 91.9 & 0.207 \\
\hline$+G N A S$ & $(2 / 3)$ & $(4 / 21)$ & 66.7 & 81.0 & 79.2 & 4 & 33.3 & 94.4 & 0.143 \\
\hline$+H C$ GNAS & $(1 / 3)$ & $(1 / 21)$ & 33.3 & 95.2 & 87.5 & 7 & 50.0 & 90.9 & 0.239 \\
\hline$+K R A S+G N A S+L O H$ & $(1 / 3)$ & $(1 / 18)$ & 33.3 & 94.4 & 85.7 & 6 & 50.0 & 89.5 & 0.271 \\
\hline$+K R A S+L O H$ & $(2 / 20)$ & $(4 / 200)$ & 10.0 & 98.0 & 90.0 & 5 & 33.3 & 91.6 & 0.094 \\
\hline High Risk DNA & - & - & - & - & - & - & - & - & - \\
\hline$+H C K R A S$ & $(3 / 22)$ & $(10 / 235)$ & 13.6 & 95.7 & 88.7 & 3 & 23.1 & 92.2 & 0.089 \\
\hline+ GNAS & $(2 / 3)$ & $(4 / 21)$ & 66.7 & 81.0 & 79.2 & 4 & 33.3 & 94.4 & 0.143 \\
\hline$+H C$ GNAS & $(1 / 3)$ & $(1 / 21)$ & 33.3 & 95.2 & 87.5 & 7 & 50.0 & 90.9 & 0.239 \\
\hline$+\mathrm{LOH}$ & $(2 / 20)$ & $(6 / 200)$ & 10.0 & 97.0 & 89.1 & 3 & 25.0 & 91.5 & 0.158 \\
\hline$+K R A S+G N A S+L O H$ & $(1 / 3)$ & $(1 / 18)$ & 33.3 & 94.4 & 85.7 & 6 & 50.0 & 89.5 & 0.271 \\
\hline$+K R A S+L O H$ & $(1 / 20)$ & $(3 / 200)$ & 5.0 & 98.5 & 90.0 & 3 & 25.0 & 91.2 & 0.319 \\
\hline$+K R A S+H C L O H$ & $(1 / 20)$ & $(0 / 200)$ & 5.0 & 100.0 & 91.4 & - & 100.0 & 91.3 & 0.091 \\
\hline KRAS & $(11 / 22)$ & $(87 / 235)$ & 50.0 & 63.0 & 61.9 & 1 & 11.2 & 93.1 & 0.231 \\
\hline+ GNAS & $(2 / 3)$ & $(4 / 21)$ & 66.7 & 81.0 & 79.2 & 4 & 33.3 & 94.4 & 0.143 \\
\hline$+\mathrm{LOH}$ & $(3 / 20)$ & $(12 / 200)$ & 15.0 & 94.0 & 86.8 & 3 & 20.0 & 91.7 & 0.144 \\
\hline HC KRAS & $(5 / 22)$ & $(33 / 235)$ & 22.7 & 86.0 & 80.5 & 2 & 13.2 & 92.2 & 0.340 \\
\hline$+H C$ GNAS & $(1 / 3)$ & $(1 / 21)$ & 33.3 & 95.2 & 87.5 & 7 & 50.0 & 90.9 & 0.239 \\
\hline GNAS & $(2 / 3)$ & $(6 / 21)$ & 66.7 & 71.4 & 70.8 & 2 & 25.0 & 93.8 & 0.249 \\
\hline HC GNAS & $(1 / 3)$ & $(2 / 21)$ & 33.3 & 90.5 & 83.3 & 4 & 33.3 & 90.5 & 0.343 \\
\hline LOH & $(4 / 20)$ & $(20 / 200)$ & 20.0 & 90.0 & 83.6 & 2 & 16.7 & 91.8 & 0.247 \\
\hline CEA $>1000$ & $(7 / 15)$ & $(58 / 209)$ & 46.7 & 72.2 & 70.5 & 2 & 10.8 & 95.0 & 0.143 \\
\hline
\end{tabular}

TP True Positive (n-positive/n-tested in malignant group); FP False Positive (n-positive/n-tested in benign group); $S n$ Sensitivity; Sp Specificity; Acc Accuracy; LR+ Positive Likelihood Ratio; PPV Positive Predictive Value; NPV Negative Predictive Value; HC LOH High Clonality Loss of Heterozygosity of Tumor Suppressor Genes; HC KRAS High Clonality KRAS Mutation; HC GNAS High Clonality GNAS Mutation; High Quantity + Quality DNA = High Risk DNA 
Table 2s. DNA Components Not Reaching Statistical Significance for HGD/Invasive Outcome:

Surgery Only

\begin{tabular}{|c|c|c|c|c|c|c|c|c|c|}
\hline & TP & FP & $\begin{array}{l}\text { Sn } \\
\text { (\%) }\end{array}$ & $\begin{array}{l}\text { Sp } \\
\text { (\%) }\end{array}$ & $\begin{array}{l}\text { Acc } \\
\text { (\%) }\end{array}$ & LR+ & $\begin{array}{l}\text { PPV } \\
(\%)\end{array}$ & $\begin{array}{l}\text { NPV } \\
\text { (\%) }\end{array}$ & $\begin{array}{c}\text { P- } \\
\text { Value }\end{array}$ \\
\hline High Quantity DNA & - & - & - & - & - & - & - & - & - \\
\hline$+K R A S$ & $(8 / 22)$ & $(16 / 67)$ & 36.4 & 76.1 & 66.3 & 2 & 33.3 & 78.5 & 0.252 \\
\hline$+H C K R A S$ & $(3 / 22)$ & $(6 / 67)$ & 13.6 & 91.0 & 71.9 & 2 & 33.3 & 76.3 & 0.684 \\
\hline+ +GNAS & $(2 / 3)$ & $(4 / 15)$ & 66.7 & 73.3 & 72.2 & 3 & 33.3 & 91.7 & 0.245 \\
\hline$+H C$ GNAS & $(1 / 3)$ & $(1 / 15)$ & 33.3 & 93.3 & 83.3 & 5 & 50.0 & 87.5 & 0.314 \\
\hline$+\mathrm{LOH}$ & $(3 / 20)$ & $(6 / 55)$ & 15.0 & 89.1 & 69.3 & 1 & 33.3 & 74.2 & 0.693 \\
\hline$+\mathrm{HC} L \mathrm{LH}$ & $(3 / 20)$ & $(2 / 55)$ & 15.0 & 96.4 & 74.7 & 4 & 60.0 & 75.7 & 0.114 \\
\hline$+K R A S+G N A S+L O H$ & $(1 / 3)$ & $(1 / 12)$ & 33.3 & 91.7 & 80.0 & 4 & 50.0 & 84.6 & 0.371 \\
\hline$+K R A S+L O H$ & $(1 / 20)$ & $(3 / 55)$ & 5.0 & 94.5 & 70.7 & 1 & 25.0 & 73.2 & 1 \\
\hline$+K R A S+H C L O H$ & $(2 / 20)$ & $(2 / 55)$ & 10.0 & 96.4 & 73.3 & 3 & 50.0 & 74.7 & 0.288 \\
\hline High Quality DNA & - & - & - & - & - & - & - & - & - \\
\hline$+K R A S$ & $(5 / 22)$ & $(6 / 67)$ & 22.7 & 91.0 & 74.2 & 3 & 45.5 & 78.2 & 0.131 \\
\hline$+H C K R A S$ & $(2 / 22)$ & $(2 / 67)$ & 9.1 & 97.0 & 75.3 & 3 & 50.0 & 76.5 & 0.254 \\
\hline$+G N A S$ & $(2 / 3)$ & $(4 / 15)$ & 66.7 & 73.3 & 72.2 & 3 & 33.3 & 91.7 & 0.245 \\
\hline$+H C$ GNAS & $(1 / 3)$ & $(1 / 15)$ & 33.3 & 93.3 & 83.3 & 5 & 50.0 & 87.5 & 0.314 \\
\hline$+\mathrm{LOH}$ & $(3 / 20)$ & $(3 / 55)$ & 15.0 & 94.5 & 73.3 & 3 & 50.0 & 75.4 & 0.333 \\
\hline$+K R A S+G N A S+L O H$ & $(1 / 3)$ & $(1 / 12)$ & 33.3 & 91.7 & 80.0 & 4 & 50.0 & 84.6 & 0.371 \\
\hline$+K R A S+L O H$ & $(2 / 20)$ & $(3 / 55)$ & 10.0 & 94.5 & 72.0 & 2 & 40.0 & 74.3 & 0.605 \\
\hline High Risk DNA & - & - & - & - & - & - & - & - & - \\
\hline$+K R A S$ & $(4 / 22)$ & $(6 / 67)$ & 18.2 & 91.0 & 73.0 & 2 & 40.0 & 77.2 & 0.255 \\
\hline$+H C K R A S$ & $(3 / 22)$ & $(6 / 67)$ & 13.6 & 91.0 & 71.9 & 2 & 33.3 & 76.3 & 0.684 \\
\hline$+G N A S$ & $(2 / 3)$ & $(4 / 15)$ & 66.7 & 73.3 & 72.2 & 3 & 33.3 & 91.7 & 0.245 \\
\hline$+H C$ GNAS & $(1 / 3)$ & $(1 / 15)$ & 33.3 & 93.3 & 83.3 & 5 & 50.0 & 87.5 & 0.314 \\
\hline$+\mathrm{LOH}$ & $(2 / 20)$ & $(3 / 55)$ & 10.0 & 94.5 & 72.0 & 2 & 40.0 & 74.3 & 0.605 \\
\hline$+K R A S+G N A S+L O H$ & $(1 / 3)$ & $(1 / 15)$ & 33.3 & 91.7 & 80.0 & 4 & 50.0 & 84.6 & 0.371 \\
\hline$+K R A S+L O H$ & $(1 / 20)$ & $(3 / 55)$ & 5.0 & 94.5 & 70.7 & 1 & 25.0 & 73.2 & 1 \\
\hline$+K R A S+H C L O H$ & $(1 / 20)$ & $(0 / 55)$ & 5.0 & 100.0 & 74.7 & - & 100.0 & 74.3 & 0.267 \\
\hline KRAS & $(11 / 22)$ & $(34 / 67)$ & 50.0 & 49.3 & 49.4 & 1 & 24.4 & 75.0 & 0.952 \\
\hline$+G N A S$ & $(2 / 3)$ & $(4 / 15)$ & 66.7 & 73.3 & 72.2 & 3 & 33.3 & 91.7 & 0.245 \\
\hline$+\mathrm{LOH}$ & $(3 / 20)$ & $(7 / 55)$ & 15.0 & 87.3 & 68.0 & 1 & 30.0 & 73.9 & 1 \\
\hline$+H C L O H$ & $(3 / 20)$ & $(3 / 55)$ & 15.0 & 94.5 & 73.3 & 3 & 50.0 & 75.4 & 0.333 \\
\hline HC KRAS & $(5 / 22)$ & $(16 / 67)$ & 22.7 & 76.1 & 62.9 & 1 & 23.8 & 75.0 & 0.912 \\
\hline$+H C$ GNAS & $(1 / 3)$ & $(1 / 15)$ & 33.3 & 93.3 & 83.3 & 5 & 50.0 & 87.5 & 0.314 \\
\hline$+\mathrm{HC} L \mathrm{OH}$ & $(3 / 20)$ & $(2 / 55)$ & 15.0 & 96.4 & 74.7 & 4 & 60.0 & 75.7 & 0.114 \\
\hline GNAS & $(2 / 3)$ & $(6 / 15)$ & 66.7 & 60.0 & 61.1 & 2 & 25.0 & 90.0 & 0.559 \\
\hline HC GNAS & $(1 / 3)$ & $(2 / 15)$ & 33.3 & 86.7 & 77.8 & 3 & 33.3 & 86.7 & 0.442 \\
\hline LOH & $(4 / 20)$ & $(10 / 55)$ & 20.0 & 81.8 & 65.3 & 1 & 28.6 & 73.8 & 1 \\
\hline CEA $>1000$ & $(7 / 15)$ & $(22 / 62)$ & 46.7 & 64.5 & 61.0 & 1 & 24.1 & 83.3 & 0.423 \\
\hline
\end{tabular}


TP True Positive (n-positive/n-tested in malignant group); FP False Positive (n-positive/n-tested in benign group); $S n$ Sensitivity; Sp Specificity; Acc Accuracy; LR+ Positive Likelihood Ratio; PPV Positive Predictive Value; NPV Negative Predictive Value; HC LOH High Clonality Loss of Heterozygosity of Tumor Suppressor Genes; HC KRAS High Clonality KRAS Mutation; HC GNAS High Clonality GNAS Mutation; High Quantity + Quality DNA = High Risk DNA 


\section{REFERENCES}

1. Ohashi K, Murakami Y, Maruyama M, et al. Four cases of mucous secreting pancreatic cancer. Prog Dig Endosc. 1982; 20: 348-351.

2. Kosmahl M, Pauser U, Peters K, Sipos B, Luttges J, Kremer B, et al. Cystic neoplasms of the pancreas and tumor-like lesions with cystic features: a review of 418 cases and a classification proposal. Virchows Arch. 2004;445:168-78.

3. Tanaka M, Fernandez-del Castillo C, Adsay V, Chari S, Falconi M, Jang JY, et al. International consensus guidelines 2012 for the management of IPMN and MCN of the pancreas. Pancreatology. 2012;12:183-97.

4. Jang JY, Park T, Lee S, Kang MJ, Lee SY, Lee KB, et al. Validation of international consensus guidelines for the resection of branch duct-type intraductal papillary mucinous neoplasms. Br J Surg. 2014;101:686-92.

5. Al-Haddad MA, Kowalski T, Siddiqui A, Mertz HR, Mallat D, Haddad N, et al. Integrated molecular pathology accurately determines the malignant potential of pancreatic cysts. Endoscopy. 2015;47:136-42.

6. Interpace Diagnostics, LLC. How PancraGEN Works [Internet]. Parsippany, NJ: Interpace Diagnostics, LLC; 2016 [cited 2018 Feb 16]. Available from: http://www.interpacediagnostics.com/pancragen/how-it-works/

7. Winner M, Sethi A, Poneros JM, Stavropoulos SN, Francisco P, Lightdale CJ, et al. The role of molecular analysis in the diagnosis and surveillance of pancreatic cystic neoplasms. Jop. 2015;16:143-9.

8. Shen J, Brugge WR, Dimaio CJ, Pitman MB. Molecular analysis of pancreatic cyst fluid: a comparative analysis with current practice of diagnosis. Cancer. 2009;117:217-27.

9. Khalid A, McGrath KM, Zahid M, Wilson M, Brody D, Swalsky P, et al. The role of pancreatic cyst fluid molecular analysis in predicting cyst pathology. Clin Gastroenterol Hepatol. 2005;3:967-73.

10. Khalid A, Zahid M, Finkelstein SD, LeBlanc JK, Kaushik N, Ahmad N, et al. Pancreatic cyst fluid DNA analysis in evaluating pancreatic cysts: a report of the PANDA study. Gastrointest Endosc. 2009;69:1095-102.

11. Rockacy MJ, Zahid M, McGrath KM, Fasanella KE, Khalid A. Association between KRAS mutation, detected in pancreatic cyst fluid, and long-term outcomes of patients. Clin Gastroenterol Hepatol. 2013;11:425-9.

12. Thiruvengadam N, Park WG. Systematic Review of Pancreatic Cyst Fluid Biomarkers: The Path Forward. Clin Transl Gastroenterol. 2015;6:e88. 
13. Kung JS, Lopez OA, McCoy EE, Reicher S, Eysselein VE. Fluid genetic analyses predict the biological behavior of pancreatic cysts: three-year experience. Jop. 2014;15:427-32.

14. Talar-Wojnarowska R, Pazurek M, Durko L, Degowska M, Rydzewska G, Smigielski J, et al. A comparative analysis of K-ras mutation and carcinoembryonic antigen in pancreatic cyst fluid. Pancreatology. 2012;12:417-20.

15. Singhi AD, Nikiforova MN, Fasanella KE, McGrath KM, Pai RK, Ohori NP, et al. Preoperative GNAS and KRAS testing in the diagnosis of pancreatic mucinous cysts. Clin Cancer Res. 2014;20:4381-9.

16. Nikiforova MN, Khalid A, Fasanella KE, McGrath KM, Brand RE, Chennat JS, et al. Integration of KRAS testing in the diagnosis of pancreatic cystic lesions: a clinical experience of 618 pancreatic cysts. Mod Pathol. 2013;26:1478-87.

17. Al-Haddad M, DeWitt J, Sherman S, Schmidt CM, LeBlanc JK, McHenry L, et al. Performance characteristics of molecular (DNA) analysis for the diagnosis of mucinous pancreatic cysts. Gastrointest Endosc. 2014;79:79-87.

18. Siddiqui AA, Kowalski TE, Kedika R, Roy A, Loren DE, Ellsworth E, et al. EUS-guided pancreatic fluid aspiration for DNA analysis of KRAS and GNAS mutations for the evaluation of pancreatic cystic neoplasia: a pilot study. Gastrointest Endosc.

2013;77:669-70.

19. Wu J, Matthaei H, Maitra A, Dal Molin M, Wood LD, Eshleman JR, et al. Recurrent GNAS mutations define an unexpected pathway for pancreatic cyst development. Sci Transl Med. 2011;3:92ra66.

20. Sawhney MS, Devarajan S, O'Farrel P, Cury MS, Kundu R, Vollmer CM, et al. Comparison of carcinoembryonic antigen and molecular analysis in pancreatic cyst fluid. Gastrointest Endosc. 2009;69:1106-10.

21. Schoedel KE, Finkelstein SD, Ohori NP. K-Ras and microsatellite marker analysis of fine-needle aspirates from intraductal papillary mucinous neoplasms of the pancreas. Diagn Cytopathol. 2006;34:605-8.

22. Interpace Diagnostics, LLC. PanDNA: Pancreatic Cyst Molecular Classifier [Internet]. Parsippany, NJ: Interpace Diagnostics, LLC; 2016 [cited 2018 Feb 16]. Available from: http://www.interpacediagnostics.com/pancragen/pandna/

23. Garud SS, Willingham FF. Molecular analysis of cyst fluid aspiration in the diagnosis and risk assessment of cystic lesions of the pancreas. Clin Transl Sci. 2012;5:102-7.

24. National Cancer Institute. What is Cancer? [Internet]. Bethesda, MD: National Cancer Institute; 2015 [cited 2018 Feb 16]. Available from: https://www.cancer.gov/aboutcancer/understanding/what-is-cancer 
25. Suzuki R, Thosani N, Annangi S, Guha S, Bhutani MS. Diagnostic yield of EUS-FNAbased cytology distinguishing malignant and benign IPMNs: a systematic review and meta-analysis. Pancreatology. 2014;14:380-4. 